\title{
Quantum self-amplified spontaneous emission regime of the x-ray Compton laser
}

\author{
H. K. Avetissian* and G. F. Mkrtchian \\ Department of Quantum Electronics, Yerevan State University, 1 A. Manukian, Yerevan 375025, Armenia
}

(Received 21 July 2006; published 13 March 2007)

\begin{abstract}
In this work we consider in general the high-gain SASE x-ray Compton FEL in the quantum regime of amplification arising from the self-consistent set of the Maxwell and relativistic quantum kinetic equations. The analytical solutions are obtained which are valid before the saturation. The quantum seed-shot noise and subsequent SASE power are calculated. A considerable increase in start-up and narrowing of the spectrum of the SASE power for amplifying $\mathrm{x}$-ray frequencies is shown compared with the classical SASE regime.
\end{abstract}

DOI: 10.1103/PhysRevSTAB.10.030703

PACS numbers: 41.60.Cr, 42.55.Vc, 13.60.Fz, 42.50.Fx

\section{INTRODUCTION}

The creation of x-ray lasers is a subject of extreme interest at the present time, and numerous projects around the world are focused on the development of so-called fourth generation light sources. The problem of creation of short-wave coherent electromagnetic radiation sources in general aspects reduces to the implementation of freeelectron lasers (FEL) [1]. Regarding the problem of the $\mathrm{x}$-ray FEL, there is an actual design currently in progress based on the FEL in the undulator [2]. It is expected that the $x$-ray FEL will operate in the so-called self-amplified spontaneous emission (SASE) regime providing coherent radiation of ultrarelativistic high brightness electron beams in the undulator [3]. The recent experimental success [4] shows the feasibility of constructing such facilities. Nevertheless, because there are no drivers or mirrors operable at X-ray wavelengths, these FEL systems operating in the SASE regime in which the initial shot noise on the electron beam is amplified over the course of propagation through a long wiggler, demand the lengths in the order of several tens to a hundred meters and electron energies up to several gigaelectron volts. To achieve the $\mathrm{x}$-ray domain with moderate relativistic electron beams (energy of electrons $\leq 50 \mathrm{MeV}$ ), the frequency of electron selfoscillation should be high enough $\sim 10^{14}-10^{15} \mathrm{~s}^{-1}$ (in undulator $10^{10} \mathrm{~s}^{-1}$ ). The latter can be realized, e.g., in the Compton backscattering scheme [5] or in the channeling radiation scheme [6]. On the other hand, for moderate relativistic electron beams as the photon wavelength moves into the deep UV and $\mathrm{x}$-ray regions, the interaction becomes quantum mechanical, i.e., quantum recoil becomes comparable to or larger than the gain bandwidth and the resonance widths due to energetic and angular spreads. Under these conditions quantum effects play an essential role [7-10]. The quantum effects are also essential if one considers the FEL versions where 1 or 2 degrees of freedom of the charged particles are quantized and the resonant enhancement of electron-photon interaction cross section

*Electronic address: avetissian@ysu.am holds. This takes place for the x-ray laser schemes based on the electron/positron beam channeling radiation in crystals [6].

The recent progress in laser technology that allows one to get laser pulses with peak intensities up to $10^{22} \mathrm{~W} / \mathrm{cm}^{2}$ [11] makes achievable the x-ray Compton backscattering scheme of FEL, with the manifestation of quantum effects. This scheme practically may appear more reasonable for the x-ray FEL due to a smaller set up requirements (particularly, using electron beams of considerably lower energies). A 3D numerical analysis of such a scheme in the classical high-gain regime of Compton backscatter FEL is reported in [12]. The quantum regime of the FEL oscillator was considered in [7]. In [8], quantum corrections for the classical high-gain single-pass FEL was examined, predicting a reduction in the gain. In [8] photon statistics of the FEL radiation was also considered. The nonlinear single-pass steady-state quantum regimes were considered in [9] for the x-ray Compton and undulator FELs in the regime when the quantum recoil is much larger than the electron beam energy spreads and electron beam momentum distribution is centered at the exact resonant condition for $\mathrm{x}$-ray emission. The operation of this quantum regime is similar to conventional atomic lasers (two momentum states are involved in the interaction process) and one can expect a new, unexplored quantum SASE regime of FEL [10]. In [10] FEL in the SASE regime quantizing the electron motion was considered, taking into account propagation effects. With the help of the numerical solution of a self-consistent set of equations, narrowing and purification of the SASE spectrum in a quantum regime was demonstrated.

In the present work, a scheme of x-ray coherent radiation generation in the quantum high gain and SASE regimes by means of a mildly relativistic high-density electron beam and a strong pump laser field is investigated. This makes it possible to achieve the quantum regime of generation at x-ray frequencies as well, due to radiation of high harmonics of Doppler-shifted pump frequencies in the strong laser field. The consideration is based on a selfconsistent set of Maxwell and relativistic quantum kinetic 
equations [9]. In this treatment, the radiation field is described as a classical Maxwell field while the lasing medium is described as a collection of Volkov-dressed electrons whose dynamic evolution is governed by the relativistic quantum kinetic equations. This semiclassical theory of x-ray FEL is capable of describing a rich variety of phenomena. However, there are some problems which require a fully quantized theory of the radiation field. For example, the photon statistics of the x-ray FEL can be properly understood only in the scope of the full quantum theory of a FEL.

The approximate analytical solutions are obtained which are valid before the saturation. These solutions show either classical or quantum regimes of generation takes place depending on the particle and pump laser beam parameters. The SASE power is calculated in the quantum regime.

The paper is organized as follows. In Sec. II we present the self-consistent set of equations which describe FEL dynamics in general and as the most effective case the hydrodynamic instability of a cold electron beam is considered. Quantum dynamics of x-ray Compton FEL in the SASE regime is considered in Sec. III. A concluding section summarizes the paper.

\section{QUANTUM HIGH-GAIN REGIME}

Consider a scheme of $\mathrm{x}$-ray radiation generation by means of a mildly relativistic high-density electron beam and a strong pump laser field. Taking into account certain dependence of harmonics radiation on the direction of particle motion with respect to the strong pump wave propagation and its polarization, we will consider arbitrary polarization of pump wave (elliptic). Thus, the given pump wave will be described by the vector potential

$$
\mathbf{A}=A_{0}\left\{\mathbf{e}_{1} \cos (\omega t-\mathbf{k r})+\mathbf{e}_{2} g \sin (\omega t-\mathbf{k r})\right\},
$$

where $\mathbf{e}_{1,2}$ are the unit polarization vectors, $k \equiv(\omega / c, \mathbf{k})$ is the four-wave vector, and $g$ is the parameter of ellipticity.

The state of a charged particle in the wave field (1) is characterized by the four-kinetic momentum or "quasimomentum," which is determined via free particle fourmomentum $p=(\mathcal{E} / c, \mathbf{p})$ and the relativistic invariant parameter of the wave intensity $\xi_{0}=e A_{0} /\left(m c^{2}\right)$ by the following equation:

$$
q=p+k \frac{m^{2} c^{2}}{4(k p)}\left(1+g^{2}\right) \xi_{0}^{2} .
$$

We assume a linearly polarized probe wave with fourvector potential

$$
A_{w}=\frac{\epsilon}{2}\left\{A_{e}(t, \mathbf{r}) e^{-i\left(\omega^{\prime} t-\mathbf{k}^{\prime} \mathbf{r}\right)}+\text { c.c. }\right\},
$$

where $A_{e}(t, \mathbf{r})$ is a slowly varying envelope, $k^{\prime}=$ $\left(\omega^{\prime} / c, \mathbf{k}^{\prime}\right)$ is the four-wave vector, and $\epsilon$ is the unit polarization four-vector $\left(\epsilon k^{\prime}\right)=0$. We will start from the selfconsistent set of equations which were derived in detail in
[9]:

$$
\begin{aligned}
\frac{\partial J(\mathbf{q})}{\partial t}+\overline{\mathbf{v}} \frac{\partial J(\mathbf{q})}{\partial \mathbf{r}}-i \Delta(s, \mathbf{q}) J(\mathbf{q}) & =\frac{e^{2} M^{2}}{4 \hbar c} A_{e} \delta F(\mathbf{q}), \\
\frac{\partial \delta F(\mathbf{q})}{\partial t}+\overline{\mathbf{v}} \frac{\partial \delta F(\mathbf{q})}{\partial \mathbf{r}} & =-\frac{1}{\hbar c}\left(A_{e}^{*} J(\mathbf{q})+A_{e} J^{*}(\mathbf{q})\right), \\
\frac{\partial A_{e}}{\partial t}+\frac{c^{2} \mathbf{k}^{\prime}}{\omega^{\prime}} \frac{\partial A_{e}}{\partial \mathbf{r}} & =\frac{4 \pi c}{\omega^{\prime}} \int J(\mathbf{q}) d \mathbf{q} .
\end{aligned}
$$

Here the quantity

$$
\delta F(\mathbf{q}, t)=F(\mathbf{q}, t)-F\left(\mathbf{q}_{f}, t\right)
$$

expresses population inversion in momentum space, $\mathbf{q}_{f}=$ $\mathbf{q}-\hbar \mathbf{k}^{\prime}+s \hbar \mathbf{k}$ is the final momentum of the electron after the absorption of $s$ photons from the pump wave and emission of one photon to probe wave, and $F(\mathbf{q})$ is the expectation value of the operator

$$
\hat{F}(\mathbf{q})=\frac{1}{(2 \pi \hbar)^{3}} \sum_{\sigma} \hat{a}_{\mathbf{q}, \sigma}^{+} \hat{a}_{\mathbf{q}, \sigma} .
$$

The creation and annihilation operators, $\hat{a}_{\mathbf{q}, \sigma}^{+}$and $\hat{a}_{\mathbf{q}, \sigma}$, associated with positive energy solutions, satisfy the anticommutation rules at equal times. The summation in Eq. (6) is carried out by polarization quantum number $\sigma$. The quantity $J(\mathbf{q}, \mathbf{r}, t)$ is the expectation value of the resonant current amplitude

$$
\begin{aligned}
J(\mathbf{q}, \mathbf{r}, t)= & \overline{\langle\hat{J}(\mathbf{q}, \mathbf{r}, t)\rangle}, \\
\hat{J}(\mathbf{q}, \mathbf{r}, t)= & -\frac{i e V}{(2 \pi \hbar)^{6}} \int d \mathbf{q}^{\prime} \sum_{\sigma^{\prime}, \sigma} \hat{a}_{\mathbf{q}^{\prime}, \sigma^{\prime}}^{+} \hat{a}_{\mathbf{q}, \sigma} \\
& \times M^{(s)}\left(\mathbf{q}^{\prime}, \sigma^{\prime} ; \mathbf{q}, \sigma\right) e^{i / \hbar\left(q^{\prime}-q-s \hbar k+\hbar k^{\prime}\right) x},
\end{aligned}
$$

where the bar denotes averaging over time and space much larger than $\left(1 / \omega^{\prime}, 1 / k^{\prime}\right), V$ is the quantization volume, and $M^{(s)}\left(\mathbf{q}^{\prime}, \sigma^{\prime} ; \mathbf{q}, \sigma\right)$ is the transition matrix element of the transversal velocity operator (see the Appendix).

In Eq. (4) $\overline{\mathbf{v}}=c^{2} \mathbf{q} / q_{0}$ is the mean velocity of the electron beam. The resonance detuning is

$$
\begin{aligned}
\hbar \Delta(s, \mathbf{q})= & \sqrt{c^{2} \mathbf{q}_{f}^{2}+m^{* 2} c^{4}}+\hbar \omega^{\prime} \\
& -\sqrt{c^{2} \mathbf{q}^{2}+m^{* 2} c^{4}}-s \hbar \omega,
\end{aligned}
$$

where

$$
m^{*}=m\left(1+\frac{1+g^{2}}{2} \xi_{0}^{2}\right)^{1 / 2}
$$

is the effective mass of the particle in the monochromatic wave. The coupling parameter $M$ in Eq. (4) is reduced to the usual calculation of a trace and in the general case of a particle-strong pump wave interaction geometry is presented in the Appendix of this work. The self-consistent set of Eq. (4) describes the FEL dynamics in general and is analogous to Maxwell-Bloch equations for conventional lasers. 
In this section we will solve the set of Eq. (4) for FEL at an arbitrary detuning of resonance. As the most effective case, the hydrodynamic instability of a cold electron beam will be considered assuming steady-state operation of FEL at which one can drop all partial time derivatives in Eq. (4). To achieve maximal Doppler shift and optimal conditions of amplification, we will also assume that the electron beam propagates along the probe wave $(O X)$ and counterpropagating pump photon beams. Consequently, the electron beam dynamics will be considered one dimensional.

Our goal is to determine the conditions under which we will have collective instability, which causes exponential growth of the probe wave. Hence, we will assume a small density perturbation for the electron beam and seek the solution of Eq. (4) in the form

$$
\delta F=\delta F_{0}\left(q_{x}\right)+\delta F_{1}\left(q_{x}, x\right) .
$$

Then in the first order by the field we will obtain the following set of linear equations:

$$
\begin{gathered}
\overline{\mathrm{v}} \frac{d J\left(x, q_{x}\right)}{d x}-i \Delta\left(s, q_{x}\right) J\left(x, q_{x}\right)=\frac{e^{2} M^{2}}{4 \hbar c} \delta F_{0}\left(q_{x}\right) A_{e}(x), \\
\frac{d A_{e}(x)}{d x}=\frac{4 \pi}{\omega^{\prime}} \int J\left(x, q_{x}\right) d q_{x}
\end{gathered}
$$

where

$$
\delta F_{0}\left(q_{x}\right)=F_{0}\left(q_{x}\right)-F_{0}\left(q_{x}-\frac{\hbar \omega^{\prime}}{c}-s \frac{\hbar \omega}{c}\right)
$$

is defined via initial distribution function $F_{0}\left(q_{x}\right)$.

Performing Laplace transformation

$$
f(\eta)=\int_{0}^{\infty} f(x) e^{-\eta x} d x
$$

for the functions $J\left(\eta, q_{x}\right)$ and $A_{e}(\eta)$, we obtain

$$
\begin{gathered}
{\left[\overline{\mathrm{v}} \eta-i \Delta\left(s, q_{x}\right)\right] J\left(\eta, q_{x}\right)=\frac{e^{2} M^{2}}{4 \hbar c} \delta F_{0}\left(q_{x}\right) A_{e}(\eta),} \\
\eta A_{e}(\eta)=\frac{4 \pi}{\omega^{\prime}} \int J\left(\eta, q_{x}\right) d q_{x} .
\end{gathered}
$$

From these equations we arrive at the following characteristic equation for variable $\eta$ :

$$
\eta=\frac{\pi e^{2} M^{2}}{\hbar \omega^{\prime} c} \int \frac{\delta F_{0}\left(q_{x}\right)}{\overline{\mathrm{v}} \eta-i \Delta\left(s, q_{x}\right)} d q_{x} .
$$

For the initial cold electron beam with the distribution function

$$
F_{0}\left(q_{x}\right)=N_{0} \delta\left(q_{x}-\bar{q}\right)
$$

( $N_{0}$ is the mean density of the electron beam) from Eq. (15), one can obtain the equation

$$
\eta=\chi^{2}\left[\frac{1}{\eta-i \frac{\Delta_{e}}{\bar{v}}}-\frac{1}{\eta-i \frac{\Delta_{a}}{\bar{v}}}\right]
$$

where

$$
\begin{gathered}
\Delta_{e}=\omega^{\prime}\left(1-\frac{\overline{\mathrm{v}}}{c}\right)-s \omega\left(1+\frac{\overline{\mathrm{v}}}{c}\right)+\frac{2 s \hbar \omega \omega^{\prime}}{\bar{q}_{0}} \\
\Delta_{a}=\Delta\left(s, \bar{q}+\frac{\hbar \omega^{\prime}}{c}+s \frac{\hbar \omega}{c}\right)=\Delta_{e}-\frac{4 s \hbar \omega \omega^{\prime}}{\bar{q}_{0}}
\end{gathered}
$$

are the resonance widths for the emission and absorption and

$$
\chi^{2}=\frac{\pi e^{2} M^{2} N_{0}}{\hbar \omega^{\prime} c \overline{\mathrm{v}}}
$$

is the main characteristic parameter of amplification in the quantum regime. Equation (17) is the characteristic equation known in the FEL theory, but it is more generalized and includes the quantum effects. We will solve the latter in two opposite limits, which characterize the quantum and classical high-gain regimes.

In the quantum regime when the electron beam momentum distribution is centered at $\Delta_{e}=0$ and

$$
|\chi| \ll \frac{4 s \hbar \omega \omega^{\prime}}{\bar{v}_{0}}
$$

the second term in the square brackets of Eq. (17) can be neglected and we obtain $\eta= \pm \chi$, whence the exponential growth rate in the quantum regime will be

$$
G_{q}=\sqrt{\frac{\pi e^{2} M^{2} N_{0}}{\hbar \omega^{\prime} c \overline{\mathrm{v}}}} .
$$

In the classical limit the quantum recoil can be neglected and since in this limit

$$
\omega^{\prime}\left(1-\frac{\overline{\mathrm{v}}}{c}\right)=s \omega\left(1+\frac{\overline{\mathrm{v}}}{c}\right)
$$

(classical resonance), Eq. (17) under the condition

$$
|\eta|^{2} \gg \frac{\Delta_{e}^{2}}{\overline{\mathrm{v}}^{2}}
$$

can be rewritten as

$$
\eta^{3}=2 i \chi^{2} \frac{\Delta_{e}}{\overline{\mathrm{v}}},
$$

whence the unstable root defines the classical result for exponential growth rate:

$$
G_{c l} \equiv \frac{\sqrt{3}}{2}\left(2 \chi^{2} \frac{\Delta_{e}}{\overline{\mathrm{v}}}\right)^{1 / 3},
$$

where $\Delta_{e}=2 s \hbar \omega^{\prime} \omega / \bar{q}_{0}$. Recalling the definition (20) for the parameter $\chi$ and using Eq. (26), the classical exponential growth rate can be written as 


$$
G_{c l} \equiv \frac{\sqrt{3}}{2}\left(s \omega \frac{4 \pi e^{2} M^{2} N_{0}}{\overline{\mathrm{v}}^{2} c \bar{q}_{0}}\right)^{1 / 3} .
$$

In particular, at the linear polarization of the pump field for the on-axis radiation from Eqs. (A11) and (A12) for the classical exponential growth rate (27), we obtain the known equation

$$
G_{c l} \equiv \frac{\sqrt{3}}{2}\left(\frac{2 s \omega \pi e^{2} N_{0}}{\overline{\mathrm{v}}^{2} m c} \frac{\xi_{0}^{2}}{\gamma_{L}^{3}} \Lambda^{2}\right)^{1 / 3}
$$

Finally, we note that the condition (24) for the classical high-gain regime can be written as

$$
\chi \gg \frac{\Delta_{e}}{\overline{\mathrm{v}}},
$$

which is opposite the condition for the quantum regime (21). As seen from Eqs. (22) and (28), the characteristic gain length of the quantum regime is scaled as $N_{0}^{-1 / 2}$ and reduces more rapidly with the increase in the beam density compared with the classical high-gain regime where gain length is scaled as $N_{0}^{-1 / 3}$.

\section{QUANTUM SASE REGIME OF FEL}

In the previous section, we have described the FEL dynamics by the universal self-consistent set of Eq. (4) which has been solved in the steady-state regime neglecting the dependence on time. Here we describe the FEL dynamics in the SASE regime taking into account the dependence on time. Thus, we will not consider diffraction or saturation effects and the FEL dynamics will be considered to be one dimensional. Taking into account the mentioned fact and keeping the time derivatives in Eq. (4), in a similar way as was done with respect to Eqs. (9) and (10) we will obtain the following set of linear equations:

$$
\begin{aligned}
& \frac{\partial J\left(x, t, q_{x}\right)}{\partial t}+\overline{\mathrm{v}} \frac{\partial J\left(x, t, q_{x}\right)}{\partial x}-i \Delta\left(s, q_{x}\right) J\left(x, t, q_{x}\right) \\
& =\frac{e^{2} M^{2}}{4 \hbar c} \delta F_{0}\left(q_{x}\right) A_{e}(x, t), \\
& \frac{\partial A_{e}(x, t)}{\partial t}+c \frac{\partial A_{e}(x, t)}{\partial x}=\frac{4 \pi c}{\omega^{\prime}} \int J\left(x, t, q_{x}\right) d q_{x},
\end{aligned}
$$

where $\delta F_{0}\left(q_{x}\right)$ is defined again by Eq. (11) via the initial distribution function of the electron beam.

By Fourier transformation for slowly varying envelopes of the probe wave and electric current density

$$
\begin{gathered}
A_{e}(x, t)=\frac{1}{2 \pi} \int_{-\infty}^{\infty} A_{\varpi}(x) e^{i \varpi t} d \varpi, \\
J\left(x, t, q_{x}\right)=\frac{1}{2 \pi} \int_{-\infty}^{\infty} J_{\varpi}\left(x, q_{x}\right) e^{i \varpi t} d \varpi,
\end{gathered}
$$

Eqs. (30) and (31) are reduced to the equations

$$
\begin{gathered}
\frac{\partial J_{\varpi}\left(x, q_{x}\right)}{\partial x}-i \Theta_{\varpi}\left(q_{x}\right) J_{\varpi}\left(x, q_{x}\right)=\frac{e^{2} M^{2}}{4 \hbar c \overline{\mathrm{v}}} A_{\varpi}(x) \delta F_{0}\left(q_{x}\right) \\
\frac{\partial A_{\varpi}(x)}{\partial x}+i \frac{\varpi}{c} A_{\varpi}(x)=\frac{4 \pi}{\omega^{\prime}} \int J_{\varpi}\left(x, q_{x}\right) d q_{x}
\end{gathered}
$$

where

$$
\Theta_{\varpi}\left(q_{x}\right)=\frac{\Delta\left(s, q_{x}\right)-\varpi}{\overline{\mathrm{v}}} .
$$

The solution of Eq. (34) can be written as

$$
\begin{aligned}
J_{\varpi}\left(x, q_{x}\right)= & J_{\varpi}\left(0, q_{x}\right) e^{i \Theta_{\varpi}\left(q_{x}\right) x}+\frac{e^{2} M^{2}}{4 \hbar c \overline{\mathrm{v}}} \\
& \times \int_{0}^{x} e^{i \Theta_{\varpi}\left(q_{x}\right)\left(x-x^{\prime}\right)} A_{\varpi}\left(x^{\prime}\right) \delta F_{0}\left(q_{x}\right) d x^{\prime} .
\end{aligned}
$$

Here it is assumed that

$$
J_{\varpi}\left(0, q_{x}\right)=\bar{J}_{\varpi} \delta\left(q_{x}-\bar{q}\right),
$$

where $\bar{J}_{\varpi}$ characterizes the shot noise in the electron beam or modulation depth for the initially modulated beam. Substituting Eq. (37) into Eq. (35), we obtain an integrodifferential equation for the phase transformed amplitude $\tilde{A}_{\varpi}(x)$ of the amplifying wave field:

$$
\begin{aligned}
& \frac{\partial \tilde{A}_{\varpi}(x)}{\partial x}+i\left(\frac{\varpi}{c}+\Theta_{\varpi}(\bar{q})\right) \tilde{A}_{\varpi}(x) \\
& =\frac{4 \pi}{\omega^{\prime}} \bar{J}_{\varpi}+\frac{\chi^{2}}{N_{0}} \iint_{0}^{x} e^{i\left(\Theta_{\varpi}\left(q_{x}\right)-\Theta_{\varpi}(\bar{q})\right)\left(x-x^{\prime}\right)} \\
& \quad \times \tilde{A}_{\varpi}\left(x^{\prime}\right) \delta F_{0}\left(q_{x}\right) d x^{\prime} d q_{x}
\end{aligned}
$$

where

$$
\tilde{A}_{\varpi}(x)=A_{\varpi}(x) e^{-i \Theta_{\varpi}(\bar{q}) x} .
$$

In the quantum regime, when condition (21) holds one can neglect the second term in Eq. (11) (which is equivalent to neglecting the absorption probability compared with the emission one) and put

$$
\delta F_{0}\left(q_{x}\right) \simeq N_{0} \delta\left(q_{x}-\bar{q}\right) ; \quad \Delta(s, \bar{q})=0
$$

in Eq. (39). Then we will obtain

$$
\frac{\partial \tilde{A}_{\varpi}(x)}{\partial x}-i\left(1-\frac{\overline{\mathrm{v}}}{c}\right) \frac{\varpi}{\overline{\mathrm{v}}} \tilde{A}_{\varpi}(x)=\frac{4 \pi}{\omega^{\prime}} \bar{J}_{\varpi}+\chi^{2} \int_{0}^{x} \tilde{A}_{\varpi}\left(x^{\prime}\right) d x^{\prime} .
$$

Performing Laplace transformation (12) on Eq. (42), we arrive at the following characteristic equation for variable $\eta$ :

$$
\eta^{2}-i\left(1-\frac{\overline{\mathrm{v}}}{c}\right) \frac{\varpi}{\overline{\mathrm{v}}} \eta-\chi^{2}=0,
$$

and the solution of Eq. (42) can be written as 


$$
\tilde{A}_{\varpi}(x)=\frac{1}{2 i \pi} \oint \frac{\eta \tilde{A}_{\varpi}(0)+\frac{4 \pi}{\omega^{\prime}} \bar{J}_{\varpi}}{\left(\eta-\eta_{1}\right)\left(\eta-\eta_{2}\right)} e^{\eta x} d \eta,
$$

where $\tilde{A}_{\varpi}(0)$ characterizes a coherent input signal. The contour integration in Eq. (44) is the result of the inverse Laplace transformation and encloses the poles which are the solutions of the characteristic equation (43):

$$
\begin{aligned}
& \eta_{1}=\frac{i}{2}\left(1-\frac{\overline{\mathrm{v}}}{c}\right) \frac{\varpi}{\overline{\mathrm{v}}}+\chi \sqrt{1-\frac{\left(1-\frac{\overline{\mathrm{v}}}{c}\right)^{2}}{4 \chi^{2}} \frac{\varpi^{2}}{\overline{\mathrm{v}}^{2}}}, \\
& \eta_{2}=\frac{i}{2}\left(1-\frac{\overline{\mathrm{v}}}{c}\right) \frac{\varpi}{\overline{\mathrm{v}}}-\chi \sqrt{1-\frac{\left(1-\frac{\overline{\mathrm{v}}}{c}\right)^{2}}{4 \chi^{2}} \frac{\varpi^{2}}{\overline{\mathrm{v}}^{2}}} .
\end{aligned}
$$

In Eq. (44) the term proportional to $\tilde{A}_{\varpi}(0)$ describes the amplification of the coherent input signal, while the second term proportional to $\bar{J}_{\varpi}$ describes either the amplification of the shot noise or coherent spontaneous emission (for the initially modulated electron beam). Since the main propose of this section is to study the amplification process without the initial seed, the first term will not be considered further. Hence, at $\tilde{A}_{\varpi}(0)=0$ Eq. (44) yields

$$
\tilde{A}_{\varpi}(x)=\frac{4 \pi}{\omega^{\prime}} \frac{\bar{J}_{\varpi}}{\eta_{1}-\eta_{2}} e^{\eta_{1} x}+\frac{4 \pi}{\omega^{\prime}} \frac{\bar{J}_{\varpi}}{\eta_{2}-\eta_{1}} e^{\eta_{2} x} .
$$

The root $\eta_{1}$ has a positive real part that gives rise to an exponentially growing term in the radiation intensity. Keeping only this term and taking into account that $\eta_{1}-$ $\eta_{2} \simeq 2 \chi$, we have

$$
\tilde{A}_{\varpi}(x)=\frac{2 \pi}{\omega^{\prime} \chi} \bar{J}_{\varpi} e^{\eta_{1} x}
$$

The spectral property of output radiation is defined by the dependence of $\eta_{1}$ on $\varpi$ and from Eq. (45) we obtain

$$
\operatorname{Re} \eta_{1} \simeq \chi-\frac{\left(1-\frac{\bar{v}}{c}\right)^{2}}{8 \chi} \frac{\varpi^{2}}{\bar{v}^{2}} \text {. }
$$

Let us calculate the average radiation power with the help of Poynting's vector and Parseval's theorem. Taking into account Eqs. (3) and (32) for the average spectral power, we obtain

$$
\frac{d P_{\varpi}(x)}{d \varpi}=\frac{S_{e} \omega^{\prime 2}}{8 \pi^{2} c \tau_{e}}\left\langle\left|\tilde{A}_{\varpi-\omega^{\prime}}(x)\right|^{2}\right\rangle,
$$

where $S_{e}$ and $\tau_{e}$ are the electron beam cross-sectional area and pulse duration, respectively. With the help of Eqs. (48) and (49) for the average spectral power, we will have

$$
\frac{d P_{\varpi}(x)}{d \varpi}=\frac{S_{e}\left\langle\left|\bar{J}_{\varpi-\omega^{\prime}}\right|^{2}\right\rangle}{2 c \tau_{e} \chi^{2}} \exp \left[-\frac{\left(\varpi-\omega^{\prime}\right)^{2}}{2 \Delta_{q}^{2}(x)}\right] e^{2 \chi x},
$$

where the spectral width in the quantum SASE regime is defined as follows:

$$
\Delta_{q}(x)=\sqrt{\frac{2 \chi}{x}} \frac{\overline{\mathrm{v}}}{1-\frac{\overline{\mathrm{v}}}{c}} .
$$

In the classical regime when condition (29) holds, the electrons have almost the same probability of absorption or emission of a photon and the net gain factor is proportional to the derivative of the momentum distribution function $F_{0}\left(q_{x}\right)$. Hence, from Eq. (11) one can put

$$
\delta F_{0}\left(q_{x}\right) \simeq \frac{\partial F_{0}\left(q_{x}\right)}{\partial q_{x}} \frac{\hbar \omega^{\prime}}{c} .
$$

For the initial cold electron beam (16) from Eq. (39) in this case we obtain

$$
\begin{aligned}
& \frac{\partial \tilde{A}_{\varpi}(x)}{\partial x}-i\left(1-\frac{\overline{\mathrm{v}}}{c}\right) \frac{\varpi}{\overline{\mathrm{v}}} \tilde{A}_{\varpi}(x) \\
& \quad=\frac{4 \pi}{\omega^{\prime}} \bar{J}_{\varpi}+i G_{c l}^{3} \int_{0}^{x}\left(x-x^{\prime}\right) \tilde{A}_{\varpi}\left(x^{\prime}\right) d x^{\prime},
\end{aligned}
$$

where $G_{c l}$ is the classical exponential growth rate (26). Without the initial seed, the solution of Eq. (54) is given as

$$
\tilde{A}_{\varpi}(x)=-i \frac{2}{\omega^{\prime}} \oint \frac{\bar{J}_{\varpi} \eta e^{\eta x}}{\left(\eta-\eta_{1}\right)\left(\eta-\eta_{2}\right)\left(\eta-\eta_{3}\right)} d \eta,
$$

where $\eta_{1,2,3}$ are the solutions of the known characteristic equation [3]

$$
\eta^{3}-i\left(1-\frac{\overline{\mathrm{v}}}{c}\right) \frac{\varpi}{\overline{\mathrm{v}}} \eta^{2}-i G_{c l}^{3}=0 .
$$

Hence, for the average spectral intensity (50) we have

$$
\frac{d P_{\varpi}(x)}{d \varpi}=\frac{S_{e}\left\langle\left|\bar{J}_{\varpi-\omega^{\prime}}\right|^{2}\right\rangle}{6 c \tau_{e} G_{c l}^{2}} \exp \left[-\frac{\left(\varpi-\omega^{\prime}\right)^{2}}{2 \Delta_{c l}^{2}(x)}\right] e^{2 G_{c l} x} .
$$

The spectral width in the classical SASE regime is defined as follows:

$$
\Delta_{c l}(x)=\sqrt{\frac{3 G_{c l}}{x}} \frac{\overline{\mathrm{v}}}{1-\frac{\overline{\mathrm{v}}}{c}} .
$$

We calculate next the shot noise power in the quantum SASE regime. For this purpose, we return to the microscopic definition of the current density operator (7). Averaging over transverse coordinates, the initial transverse current density operator will be represented in the form

$$
\begin{aligned}
\hat{J}(x, 0)= & \frac{e V}{i(2 \pi \hbar)^{6} S_{e}} \int d y d z d \mathbf{q}^{\prime} d \mathbf{q} \sum_{\sigma^{\prime}, \sigma} \hat{a}_{\mathbf{q}^{\prime}, \sigma^{\prime}}^{+}(0) \hat{a}_{\mathbf{q}, \sigma}(0) \\
& \times M^{(s)}\left(\mathbf{q}^{\prime}, \sigma^{\prime} ; \mathbf{q}, \sigma\right) e^{-(i / \hbar)\left(\mathbf{q}^{\prime}-\mathbf{q}-s \hbar \mathbf{k}+\hbar \mathbf{k}^{\prime}\right) \mathbf{r}} .
\end{aligned}
$$

Making a Fourier expansion in Eq. (59),

$$
\hat{J}(\kappa, 0)=\int \hat{J}(x, 0) e^{-\kappa x} d x,
$$

and integrating over $x, y, z$, and $q^{\prime}$ we obtain 


$$
\begin{aligned}
\hat{J}(\kappa, 0)= & \frac{e V}{i(2 \pi \hbar)^{3} S_{e}} \\
& \times \int d \mathbf{q} \sum_{\sigma^{\prime}, \sigma} \hat{a}_{\mathbf{q}_{r}, \sigma^{\prime}}^{+}(0) \hat{a}_{\mathbf{q}, \sigma}(0) M^{(s)}\left(\mathbf{q}_{r}, \sigma^{\prime} ; \mathbf{q}, \sigma\right),
\end{aligned}
$$

The quantity $\left\langle\left|\bar{J}_{\varpi}\right|^{2}\right\rangle$ in Eq. (51) can be defined as

$$
\left\langle\left|\bar{J}_{\varpi}\right|^{2}\right\rangle=\frac{1}{c^{2}}\left\langle\hat{J}^{+}\left(\frac{\varpi}{c}, 0\right) \hat{J}\left(\frac{\varpi}{c}, 0\right)\right\rangle .
$$

With the help of Eq. (61) we obtain

where

$$
\begin{aligned}
q_{r x}=q_{x}-s \frac{\hbar \omega}{c}-\frac{\hbar \omega^{\prime}}{c}-\hbar \kappa, \quad q_{r y}=q_{y}, q_{r z}=q_{z} \cdot \\
\left\langle\left|\bar{J}_{\varpi}\right|^{2}\right\rangle=\frac{e^{2}}{c^{2} S_{e}^{2}} \frac{V^{2}}{(2 \pi \hbar)^{6}} \int d \mathbf{q} d \mathbf{q}_{1} \sum_{\sigma_{1}^{\prime}, \sigma_{1}} \sum_{\sigma^{\prime}, \sigma}\left\langle\hat{F}_{(4)}\right\rangle M^{*(s)}\left(\mathbf{q}_{1 r}, \sigma_{1}^{\prime} ; \mathbf{q}_{1}, \sigma_{1}\right) M^{(s)}\left(\mathbf{q}_{r}, \sigma^{\prime} ; \mathbf{q}, \sigma\right),
\end{aligned}
$$

where

$$
\hat{F}_{(4)}=\hat{a}_{\mathbf{q}_{1}, \sigma_{1}}^{+}(0) \hat{a}_{\mathbf{q}_{1 r}, \sigma_{1}^{\prime}}(0) \hat{a}_{\mathbf{q}_{r}, \sigma^{\prime}}^{+}(0) \hat{a}_{\mathbf{q}, \sigma}(0) .
$$

Taking into account the anticommutation rule,

$$
\hat{a}_{\mathbf{q}_{1 r}, \sigma_{1}^{\prime}} \hat{a}_{\mathbf{q}_{r}, \sigma^{\prime}}^{+}=\frac{(2 \pi \hbar)^{3}}{V} \delta_{\sigma^{\prime} \sigma_{1}^{\prime}} \delta\left(\mathbf{q}_{1}-\mathbf{q}\right)-\hat{a}_{\mathbf{q}_{r}, \sigma^{\prime}}^{+} \hat{a}_{\mathbf{q}_{1}, \sigma_{1}^{\prime}},
$$

for the expectation value of $\hat{F}_{(4)}$ we obtain

$$
\begin{aligned}
\left\langle\hat{F}_{(4)}\right\rangle= & \frac{(2 \pi \hbar)^{3}}{V} \delta_{\sigma^{\prime} \sigma_{1}^{\prime}} \delta\left(\mathbf{q}_{1}-\mathbf{q}\right)\left\langle\hat{a}_{\mathbf{q}_{1}, \sigma_{1}}^{+}(0) \hat{a}_{\mathbf{q}, \sigma}(0)\right\rangle \\
\left\langle\hat{F}_{(4)}\right\rangle= & \frac{(2 \pi \hbar)^{3}}{V} \delta_{\sigma^{\prime} \sigma_{1}^{\prime}} \delta\left(\mathbf{q}_{1}-\mathbf{q}\right)\left\langle\hat{a}_{\mathbf{q}_{1}, \sigma_{1}}^{+}(0) \hat{a}_{\mathbf{q}, \sigma}(0)\right\rangle \\
& -\left\langle\hat{a}_{\mathbf{q}_{1}, \sigma_{1}}^{+}(0) \hat{a}_{\mathbf{q}_{r}, \sigma^{\prime}}^{+}(0) \hat{a}_{\mathbf{q}_{1}, \sigma_{1}^{\prime}}(0) \hat{a}_{\mathbf{q}, \sigma}(0)\right\rangle .
\end{aligned}
$$

The term $\left\langle\hat{a}_{\mathbf{q}_{1}, \sigma_{1}}^{+}(0) \hat{a}_{\mathbf{q}, \sigma}(0)\right\rangle$ in Eq. (63) is the single-particle density matrix:

$$
\left\langle\hat{a}_{\mathbf{q}_{1}, \sigma_{1}}^{+}(0) \hat{a}_{\mathbf{q}, \sigma}(0)\right\rangle=\rho_{\sigma \sigma_{1}}\left(\mathbf{q}, \mathbf{q}_{1}, 0\right)=\rho_{0}\left(\mathbf{q}, \mathbf{q}_{1}\right) \delta_{\sigma, \sigma_{1}} .
$$

Here it was assumed that the electron beam is nonpolarized. The density matrix $\rho_{0}(q, q)$ is connected to the classical momentum distribution function $F(q)$ by the formula

$$
\rho_{0}(\mathbf{q}, \mathbf{q})=\frac{(2 \pi \hbar)^{3}}{2} F_{0}(\mathbf{q})
$$

The second term in Eq. (63) is connected to the twoparticle density matrix and it is negligible for the nonmodulated electron beam. Hence, neglecting the second term in Eq. (63), from Eq. (62) we obtain

$$
\begin{aligned}
\left\langle\left|\bar{J}_{\varpi}\right|^{2}\right\rangle= & \frac{e^{2} V}{2 c^{2} S_{e}^{2}} \int d \mathbf{q} F_{0}(\mathbf{q}) \\
& \times \sum_{\sigma^{\prime}, \sigma} M^{*(s)}\left(\mathbf{q}_{r}, \sigma^{\prime} ; \mathbf{q}, \sigma\right) M^{(s)}\left(\mathbf{q}_{r}, \sigma^{\prime} ; \mathbf{q}, \sigma\right) .
\end{aligned}
$$

Taking into account Eq. (16), we have

$$
\left\langle\left|\bar{J}_{\varpi}\right|^{2}\right\rangle=\frac{e^{2} M^{2} V N_{0}}{2 c^{2} S_{e}^{2}},
$$

where

$$
M^{2}=\sum_{\sigma^{\prime}, \sigma} M^{*(s)}\left(\overline{\mathbf{q}}_{r}, \sigma^{\prime} ; \overline{\mathbf{q}}, \sigma\right) M^{(s)}\left(\overline{\mathbf{q}}_{r}, \sigma^{\prime} ; \overline{\mathbf{q}}, \sigma\right) .
$$

Because of the quantum nature of radiation, the expression (A9) for $M^{2}$ differs from the classical one only by the terms of quantum recoil, which are of the order of $\hbar \omega^{\prime} / \bar{q}_{0}$. Taking into account that for a FEL the condition $\hbar \omega^{\prime} / \bar{q}_{0} \ll$ 1 is always satisfied, one can omit these terms. Hence, Eq. (65) is reduced to the classical formula.

For the average spectral power in the quantum SASE regime from Eqs. (51) and (65) we will have

$$
\frac{d P_{\varpi}(x)}{d \varpi}=\frac{\hbar \omega^{\prime}}{4 \pi} \frac{\overline{\mathrm{v}}^{2}}{c^{2}} \exp \left[-\frac{\left(\varpi-\omega^{\prime}\right)^{2}}{2 \Delta_{q}^{2}(x)}\right] e^{2 \chi x} .
$$

The average total power can be obtained by integrating Eq. (67) over $\varpi$ :

$$
P(x)=\int_{0}^{\infty} \frac{d P_{\varpi}(x)}{d \varpi} d \varpi .
$$

Taking into account Eq. (52), we obtain total power in the quantum SASE regime

$$
P(\bar{x})=\frac{\hbar \omega^{\prime} \bar{\gamma}^{2} c}{L_{q}} \frac{e^{\bar{x}}}{\sqrt{2 \pi \bar{x}}} .
$$

Hear $L_{q}=1 / \chi$ is the characteristic quantum amplification length, $\bar{x}=2 x / L_{q}$ is the normalized amplification length, and $\bar{\gamma}=\bar{q}_{0} /\left(m^{*} c^{2}\right)$ is the effective Lorentz factor of the particle in the monochromatic wave [in Eq. (68) we put $\left.\overline{\mathrm{v}} \approx c, 1-\overline{\mathrm{v}} / c \approx 1 /\left(2 \bar{\gamma}^{2}\right)\right]$.

The exponential growth cannot continue indefinitely and must saturate at a certain level. The saturation effect is due to nonlinear effects and for the quantum regime it can be understood as follows. In the quantum regime the quantum recoil moves the electrons off resonance after the emission 
of a photon, thus the probability of emitting additional photons becomes negligible. Therefore the saturated power can be estimated to be

$$
P_{\mathrm{sat}} \simeq \hbar \omega^{\prime} \frac{I}{e},
$$

where $I$ is the electron beam current. Note that this result is also obtained with the nonlinear steady-state solution [9] where maximal intensity change is $-\hbar \omega^{\prime} \overline{\mathrm{v}} N_{0}$.

As is seen from conditions (21) and (24), to achieve the quantum high-gain regime, for a given probe wave frequency and electron beam energy [i.e. $\bar{q}_{0} /\left(\hbar \omega^{\prime}\right)=$ const] one should decrease electron beam density (or pump wave power). On the other hand, this enlarges the characteristic gain length. Comparing Eq. (57) with its quantum counterpart (51), it is easy to see that due to the longer gain length the spectrum of the quantum SASE power is narrowed by the factor $\Delta_{q}\left(L_{q}\right) / \Delta_{c l}\left(L_{c l}\right) \sim \chi\left(\mu_{q}\right) / G_{c l}\left(\mu_{c l}\right) \ll 1$ [see Eqs. (58) and (52)] and for the same initial shot noise in the quantum regime the start-up intensity is enhanced by the factor $G_{c l}^{2}\left(\mu_{c l}\right) / \chi^{2}\left(\mu_{q}\right) \gg 1$. Here $\mu_{c l}$ and $\mu_{q}$ are the set of parameters for classical and quantum regimes, respectively.

Note that the longer quantum gain length and, consequently, narrower line bandwidth, as well as the quantum nature of electrons' dynamics, may be the reason for spectrum purification [10] compared to classical spectrum with the spiking behavior [13]. Thus, the longer quantum gain length besides the spectrum narrowing results to more coherent seed for the downstream part of amplification. In addition, the operation of the quantum regime for Compton FEL is similar to conventional lasers (two quantum states are involved in the interaction process) and with a more coherent seed one can expect spectrum purification. The latter was demonstrated in [10] with the help of a numerical solution of a self-consistent set of equations.

For the quantum regime it is necessary to take into account the conditions [9] as well:

$\Delta_{e}-\Delta_{a}=\frac{4 s \hbar \omega \omega^{\prime}}{\bar{q}_{0}}>\max \left\{\left|\frac{\partial \Delta_{e}}{\partial \eta_{i}} \delta \eta_{i}+\frac{1}{2} \frac{\partial^{2} \Delta_{e}}{\partial \eta_{i}^{2}}\left(\delta \eta_{i}\right)^{2}\right|\right\}$,

where by $\eta_{i}$ we denote the set of quantities characterizing the electron beam and pump field, and by $\delta \eta_{i}$ their spreads. The conditions (70) and (21) can be written as

$$
\frac{\hbar \omega^{\prime}}{\bar{q}_{0}}>\max \left\{\frac{\lambda^{\prime}}{L_{q}} \frac{\bar{\gamma}^{2}}{2 \pi}, \frac{\Delta \gamma_{L}}{\gamma_{L}}, \frac{(\Delta \vartheta)^{2} \bar{\gamma}^{2}}{2}, \frac{\Delta \omega}{2 \omega}\right\} .
$$

Here

$$
\lambda^{\prime}=\frac{\lambda}{4 s \gamma_{L}^{2}}\left(1+\frac{1+g^{2}}{2} \xi_{0}^{2}\right)
$$

is the wavelength of the probe wave. The first term in the curly brackets of Eq. (71) expresses the condition (21) for quantum high gain regime. The second and third terms are the resonance widths due to energetic and angular spreads, and the last term expresses the resonance width caused by the nonmonochromaticity of the pump wave.

\section{CONCLUSION}

We have presented a theoretical treatment of quantum high-gain and SASE regimes of the x-ray Compton FEL by means of a mildly relativistic high-density electron beam and a strong pump laser field. The analytical solutions of the self-consistent set of Maxwell and relativistic quantum kinetic equations were obtained for these quantum regimes of x-ray amplification. The formula for SASE power in the quantum regime was obtained taking into account the intensity effect of strong laser field in the quantum seed exactly. This takes into account the possibility to achieve the quantum regime of generation at x-ray frequencies due to radiation of high harmonics of Doppler-shifted pump frequencies in the strong laser field. Comparisons with the classical regimes were made. The analysis of obtained results shows that in the quantum regime the start-up intensity is enhanced and the spectrum of the SASE intensity is narrowed compared with the classical SASE regime.

To specify the requirements on the particle and pump laser beams for experimental realization of the quantum SASE regime, one needs to solve the $3 \mathrm{D}$ issue which should take into account electron beam emittance, laser diffraction, and space charge effects. However, this is impossible in the scope of analytical treatment and require numerical simulations. This issue is currently under investigation and will be the subject of future work.

\section{ACKNOWLEDGMENTS}

This work was supported by International Science and Technology Center (ISTC) Project No. A-1307.

\section{APPENDIX}

In this Appendix we present general expressions for the transition matrix element of transversal velocity operator and main coupling parameter $M$ in the self-consistent set of equations (4). The transition matrix element of the transversal velocity operator for the general geometry of a particle-strong pump wave interaction with the fields (1) and (3) is given by the following formula:

$$
\begin{aligned}
M^{(s)}\left(q_{f}, \sigma^{\prime} ; q, \sigma\right)= & \frac{\bar{u}_{\sigma^{\prime}}\left(p_{f}\right)}{2 \sqrt{q_{f 0} q_{0}}}\left\{\frac{e^{2}(k \epsilon) Q_{2 s}}{2 c^{2}\left(k q_{f}\right)(k q)} \hat{k}+\hat{\epsilon} Q_{0 s}\right. \\
& \left.+\left(\frac{e \hat{Q}_{1 s} \hat{k} \hat{\epsilon}}{2 c\left(k q_{f}\right)}+\frac{e \hat{\epsilon} \hat{k} \hat{Q}_{1 s}}{2 c(k q)}\right)\right\} u_{\sigma}(p),
\end{aligned}
$$

where $u_{\sigma}$ is the bispinor amplitude of a free Dirac particle with polarization $\sigma$, the operator notation is used: $\hat{a}=$ $\gamma^{\mu} a_{\mu}$, and $\gamma^{\mu}$ are the Dirac matrices. The vector functions 
$Q_{1 s}^{\mu}=\left(0, \mathbf{Q}_{1 s}\right)$ and scalar functions $Q_{0 s}, Q_{2 s}$ are expressed via the generalized Bessel function $G_{s}(\alpha, \beta, \varphi)$ :

$$
\begin{gathered}
Q_{0 s}=G_{s}(\alpha, \beta, \varphi), \\
\mathbf{Q}_{1 s}=\frac{A_{0}}{2}\left\{\mathbf{e}_{1}\left[G_{s-1}(\alpha, \beta, \varphi)+G_{s+1}(\alpha, \beta, \varphi)\right]\right. \\
\left.+i \mathbf{e}_{2} g\left[G_{s-1}(\alpha, \beta, \varphi)-G_{s+1}(\alpha, \beta, \varphi)\right]\right\}, \\
Q_{2 s}=A_{0}^{2} \frac{\left(1+g^{2}\right)}{2} G_{s}(\alpha, \beta, \varphi)+A_{0}^{2} \frac{\left(1-g^{2}\right)}{2} \\
\quad \times\left[G_{s-2}(\alpha, \beta, \varphi)+G_{s+2}(\alpha, \beta, \varphi)\right] .
\end{gathered}
$$

The arguments $\alpha, \beta$, and $\varphi$ are

$$
\begin{gathered}
\alpha=\frac{m c \xi_{0}}{\hbar}\left[\left(\frac{\mathbf{e}_{1} \mathbf{q}}{(q k)}-\frac{\mathbf{e}_{1} \mathbf{q}_{f}}{\left(q_{f} k\right)}\right)^{2}+g^{2}\left(\frac{\mathbf{e}_{2} \mathbf{q}}{(q k)}-\frac{\mathbf{e}_{2} \mathbf{q}_{f}}{\left(q_{f} k\right)}\right)^{2}\right]^{1 / 2}, \\
\beta=\frac{m^{2} c^{2}}{8 \hbar} \xi_{0}^{2}\left(1-g^{2}\right)\left(\frac{1}{(q k)}-\frac{1}{\left(q_{f} k\right)}\right), \\
\tan \varphi=\frac{g\left(\frac{\mathbf{e}_{2} \mathbf{q}}{(q k)}-\frac{\mathbf{e}_{2} \mathbf{q}_{f}}{\left(q_{f} k\right)}\right.}{\left(\frac{\mathbf{e}_{1} \mathbf{q}}{(q k)}-\frac{\left.\mathbf{e}_{1} \mathbf{q}_{f}\right)}{\left(q_{f} k\right)}\right.} .
\end{gathered}
$$

The generalized Bessel function is expressed by the series of ordinary Bessel function productions:

$$
G_{s}(\alpha, \beta, \varphi)=\sum_{k=-\infty}^{\infty} J_{2 k-s}(\alpha) J_{k}(\beta) e^{i(s-2 k) \varphi} .
$$

The quantity $M$ [see Eq. (66)] is reduced to the usual calculation of a trace [9] and in the general case is given by the following formula:

$$
\begin{aligned}
M^{2}= & \frac{2 c^{4}}{q_{f 0} q_{0}}\left\{\left|\left[\left(p \epsilon^{\prime}\right) Q_{0 s}-\frac{e}{c}\left(Q_{1 s} \epsilon^{\prime}\right)\right]\right|^{2}\right. \\
& \left.-\frac{e^{2}}{4 c^{2}} \frac{\left(\hbar k^{\prime} k\right)^{2}}{(q k)\left(q_{f} k\right)}\left[\left|Q_{1 s}\right|^{2}+\operatorname{Re}\left(Q_{2 s} Q_{0 s}^{*}\right)\right]\right\},
\end{aligned}
$$

where

$$
\epsilon^{\prime}=\epsilon-k^{\prime} \frac{(k \epsilon)}{\left(k k^{\prime}\right)}
$$

In Eq. (A9), one can neglect the terms on the order of $\left[\hbar k^{\prime} k /(k q)\right]^{2} \ll 1$ since for a FEL this condition is always satisfied. In particular, at the linear polarization of the pump field $(g=0)$ for the on-axis radiation from these equations, we have

$$
M^{2}=c^{2} \frac{\xi_{0}^{2}}{2 \gamma_{L}^{2}} \Lambda^{2}
$$

where

$$
\Lambda=J_{(s+1) / 2}\left(\frac{s \xi_{0}^{2}}{4+2 \xi_{0}^{2}}\right)-J_{(s-1) / 2}\left(\frac{s \xi_{0}^{2}}{4+2 \xi_{0}^{2}}\right)
$$

and $\gamma_{L}$ is the Lorentz factor.

[1] T. C. Marshall, Free Electron Lasers (MacMillan, London, 1985); C. A. Brau, Free-Electron Lasers (Academic, Boston, 1990); W. B. Colson, C. Pellegrini, and A. Renieri, Laser Handbook (Elsevier, Amsterdam, 1990), Vol. 6; G. Dattoli, A. Renieri, and A. Torre, Lectures on the Free Electron Laser Theory and Related Topics (World Scientific, London, 1993); H.P. Freund and T.M. Antonsen, Jr., Principles of Free-Electron Lasers (Chapman and Hall, London, 1996); E. L. Saldin, E. A. Schneidmiller, and M. V. Yurkov, The Physics of Free Electron Lasers (Springer, Berlin, 2000).

[2] DESY Report No. 1997-048, edited by R. Brinkmann, G. Materlik, J. Rossbach, and A. Wagner, 1997; J. Arthur et al., Report No. SLAC-R-521, 1998.

[3] A. M. Kondratenko and E. L. Saldin, Part. Accel. 10, 207 (1980); R. Bonifacio, C. Pellegrini, and L. M. Narducci, Opt. Commun. 50, 373 (1984); K. J. Kim, Phys. Rev. Lett. 57, 1871 (1986); S. Krinsky and L. H. Yu, Phys. Rev. A 35, 3406 (1987).

[4] J. Andruszkow et al., Phys. Rev. Lett. 85, 3825 (2000); V. Ayvazyan et al., ibid. 88, 104802 (2002); V. Ayvazyan et al., Eur. Phys. J. D 20, 149 (2002).

[5] R. H. Pantell, G. Soncini, and H.E. Puthoff, IEEE J. Quantum Electron. 4, 905 (1968).

[6] H. K. Avetissian, K. Z. Hatsagortsian, G. F. Mkrtchian, and Kh. V. Sedrakian, Phys. Rev. A 56, 4121 (1997); H. K. Avetissian and G. F. Mkrtchian, Nucl. Instrum. Methods Phys. Res., Sect. A 507, 479 (2003).

[7] E. M. Belenov, S. V. Grigorev, A. V. Nazarkin, and I. V. Smetanin, J. Exp. Theor. Phys. 78, 431 (1994).

[8] C. B. Schroeder, C. Pellegrini, and P. Chen, Phys. Rev. E 64, 056502 (2001).

[9] H. K. Avetissian and G.F. Mkrtchian, Phys. Rev. E 65, 046505 (2002); Nucl. Instrum. Methods Phys. Res., Sect. A 483, 548 (2002).

[10] R. Bonifacio, N. Piovella, and G. R. M. Robb, Nucl. Instrum. Methods Phys. Res., Sect. A 543, 645 (2005); R. Bonifacio, N. Piovella, G. R. M. Robb, and M. M. Cola, Opt. Commun. 252, 381 (2005); R. Bonifacio, M. M. Cola, N. Piovella, and G. R. M. Robb, Europhys. Lett. 69, 55 (2005); R. Bonifacio, N. Piovella, G. R. M. Robb, and A. Schiavi, Phys. Rev. ST Accel. Beams 9, 090701 (2006).

[11] M. D. Perry, D. Pennington, B. C. Stuart, G. Tietbohl, J. A. Britten, C. Brown, S. Herman, B. Golick, M. Kartz, J. Miller, H. T. Powell, M. Vergino, and V. Yanovsky, Opt. Lett. 24, 160 (1999).

[12] A. Bacci, M. Ferrario, C. Maroli, V. Petrillo, and L. Serafini, Phys. Rev. ST Accel. Beams 9, 060704 (2006).

[13] R. Bonifacio, L. De Salvo, P. Pierini, N. Piovella, and C. Pellegrini, Phys. Rev. Lett. 73, 70 (1994). 